\title{
Research Square \\ Transport in Fusion Plasmas: Is the Tail Wagging the Dog?
}

Guilhem Dif-Pradalier ( $\sim$ difpradalier@yahoo.fr)

CEA https://orcid.org/0000-0003-4869-7049

Philippe Ghendrih

CEA

Yanick Sarazin

CEA

Elisabetta Caschera

CEA

Frederic Clairet

CEA

Yann Camenen

CNRS

Xavier Garbet

CEA

Virginie Grandgirard

CEA

Yann Munschy

CEA

Laure Vermare

CNRS

Fabien Widmer

CNRS

\section{Physics Article}

Keywords: fusion plasmas, turbulent plasmas

Posted Date: September 8th, 2021

DOI: https://doi.org/10.21203/rs.3.rs-879691/v1

License: (a) (i) This work is licensed under a Creative Commons Attribution 4.0 International License.

Read Full License 
Version of Record: A version of this preprint was published at Communications Physics on September 15th, 2022. See the published version at https://doi.org/10.1038/s42005-022-01004-z. 
Transport in Fusion Plasmas: Is the Tail Wagging the Dog?

G. Dif-Pradalier ${ }^{1, *}$, Ph. Ghendrih ${ }^{1}$, Y. Sarazin ${ }^{1}$, E. Caschera ${ }^{1}$, F. Clairet ${ }^{1}$, Y. Camenen ${ }^{2}$, X. Garbet ${ }^{1}$, V. Grandgirard $^{1}$, Y. Munschy' ${ }^{1}$, L. Vermare ${ }^{3}$, F. Widmer ${ }^{2}$

1) CEA, IRFM, F-13108 Saint-Paul-lez-Durance cedex, France

2) CNRS, Aix-Marseille Univ., PIIM UMR7345, Marseille, France

3) L.P.P. Ecole Polytechnique, Palaiseau, France

*e-mail: guilhem.dif-pradalier@cea.fr

Turbulent plasmas notably self-organize to higher energy states upon application of additional free energy sources or modification of edge operating conditions. Mechanisms whereby such bifurcations occur have been actively debated for decades, mostly on the basis of reduced models. Here we unravel a surprising causal chain of events in the onset of an improved confinement state, by applying generic entropy-based and information theoretic measures to the primitive kinetic equations. Interfacial contamination of a large, stable region (the 'dog') by locally-borne peripheral turbulent fluctuations (the 'tail') is found to be central to explaining transport properties, globally. These results, highly relevant to the quest for magnetic fusion advocate the use of such data-driven methods to many problems in fluids and plasmas where interfacial turbulent contamination is active.

\section{Main text}

When observed, the origin of turbulence activity somewhere prompts speculation. The base state may be unstable. Turbulent fluctuations may also have originated elsewhere and expanded beyond the region of convective drive. Interfacial contamination by turbulence is generic in fluids and plasmas, referred to as nibbling (1) or engulfment (2) in jet interfaces, overshooting or penetration (3) in geophysical and astrophysical fluids or spreading (4-6) in magnetized plasmas. Subcritical transitions to turbulence in parallel flows have been evidenced, belonging to the class of directed percolation (7). As turbulence drive flows which feedback on transport, clarifying the precise mechanisms whereby a system may transition to and sustain a turbulent state is of great theoretical and practical importance.

Magnetized fusion plasmas are oft separated between a hot confined core region where fusion occurs, an unconfined peripheral boundary layer (the Scrape-Off Layer or SOL) and an inbetween edge region, loosely defined, set between core and separatrix. The SOL is cold and comparatively rarefied, starting at the magnetic separatrix and magnetically connected to the material boundaries. Core and SOL have been extensively studied, mostly independently, the edge usually serving as fixed boundary condition for both, its dynamics difficult to apprehend. 
This separation is increasingly being questioned. Tokamak plasmas are indeed prone to selforganization, a prominent example being the spontaneous transition between the lowconfinement "L-mode" to high-confinement "H-mode" (8). The H-mode branch of operation is one of several improved confinement states that have been experimentally discovered, revitalizing the fusion program towards ITER. Significant performance enhancement has been made possible by exploiting the spontaneous $\mathrm{L}$ to $\mathrm{H}$ transition, induced by increased radial electric field shear Er'. Onset of differential rotation, which scales with Er' and steepening of the ion pressure profile in a localized region of the L-mode edge -the so-called "pedestal"stabilizes turbulence, reduces transport and initiates a self-reinforcing feedback $(9,10)$ which locks-in the bifurcated state. Comprehensive description of this dynamics from first principles is still lacking and certainly requires the intermediate step of apprehending turbulence dynamics in the L-mode edge, from where the bifurcation originates.

The L-mode edge is challenging from a modelling standpoint. Relative turbulence fluctuations levels $\delta n / n, n$ being the plasma density are experimentally observed to increase from core to separatrix (11). At the same time, large fractions of the outer edge are either linearly stable or marginally unstable to drift-wave interchange turbulence, questioning the origin of observed fluctuations there. Hence its oft-used name of "No Man's Land" (NMsL) bearing witness to the difficult understanding of its dynamics. The incentives to clarify this problem are high for turbulence activity in the edge controls stress and electric field dynamics, affecting access to transport bifurcated states and deposition patterns on plasma facing components, highly relevant to the safe operation of large burning plasma systems.

Turbulent scales at the plasma edge are comparable to free energy gradient scales and oftassumed separations between a slow, large-scale background and fast, small-scale fluctuations become inadequate, as already noted by Kadomtsev (12). Two modelling strategies are commonly followed: numerically-efficient mixing-length inspired "gradient-driven" models, initially developed for the confined core impose such scale separations through fixed mean gradients whilst "flux-driven" models, as in experiments prescribe adiabatic sources and sinks and compute dynamically-evolving profiles, flows, stresses and fluxes. Owing to complexity and computational cost, flux-driven gyrokinetic (13) investigations of the L-mode edge are scarce. This work proposes a comprehensive discussion from the primitive kinetic equations both in flux-driven and gradient-driven regimes of the mechanisms whereby turbulence organizes in the plasma edge when core, edge and SOL may dynamically interplay. Notably, 
we find that the elusive questions of stability of NMsL and the sought-after onset of an edge transport barrier are intimately connected. Key results are: (i) boundary-mediated interactions trigger spontaneous symmetry breaking and localized destabilization of the outer plasma edge. Constant and localized turbulence intensity production follows, which (ii) spreads, contaminating stable NMsL on global scales. Concomitant to turbulence production, (iii) a persistent transport barrier emerges, at nominal experimental parameters. Flows driven by the density and temperature gradients, dubbed « diamagnetic » are found to be surprisingly influential to edge transport barrier onset, acting through seldom-invoked mechanisms. At last, (iv) removal of the plasma-boundary interface or postulation of scale separations hinders turbulence spreading, resulting in a laminar edge which prevents access to edge transport barrier onset.
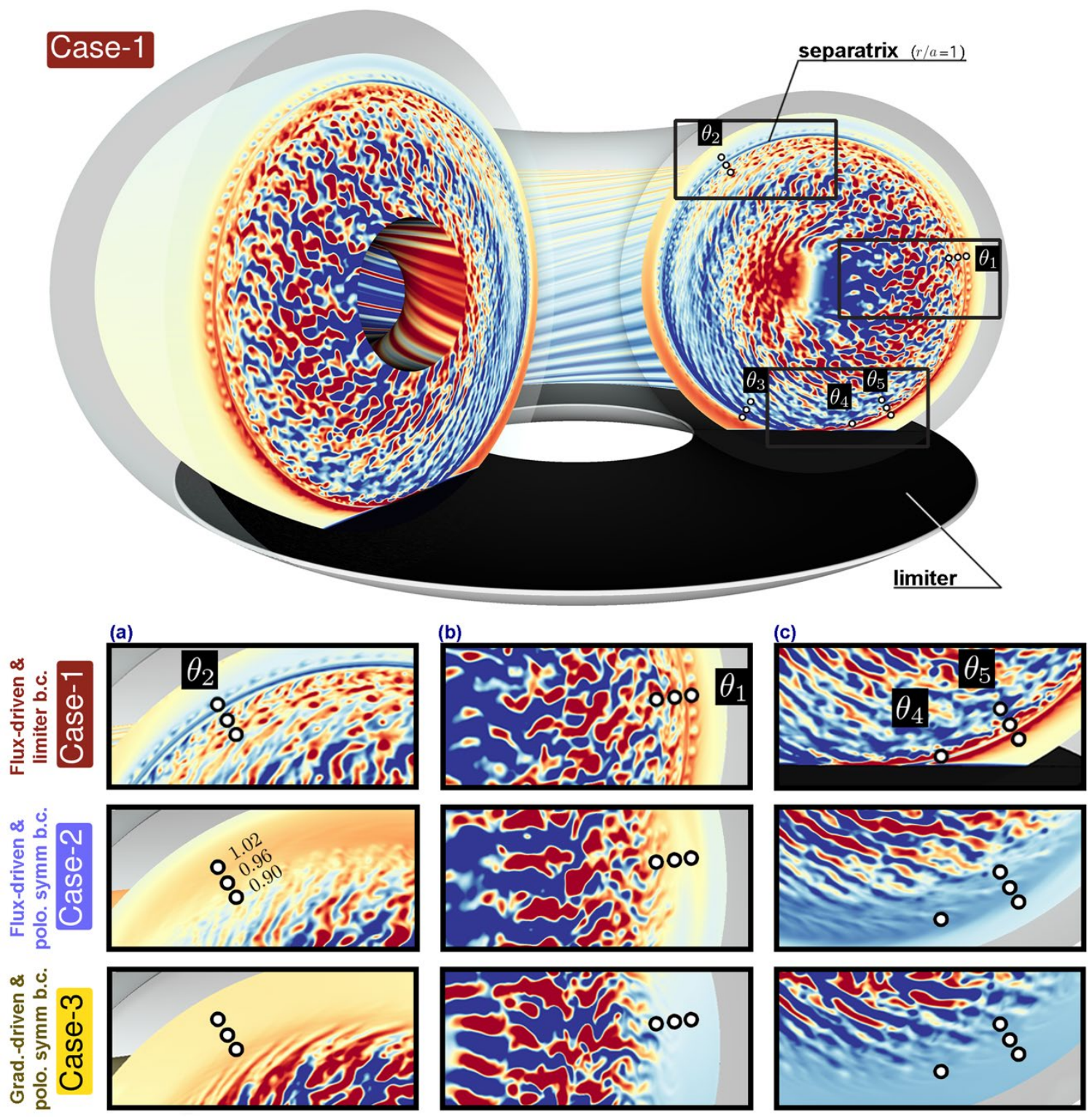
Fig. 1. Snapshots of the electrostatic potential in three different configurations, at statistical equilibrium. Case-1 is the reference flux-driven configuration and features, as in experiments, a transition from closed to open field lines through introduction of a poloidally-localized toroidal limiter (separatrix is at normalized radius $\rho=r / a=1$ ) and a wall (at $\rho=1.3$ ) within the computational domain. Case-2 differs from Case-1 through its external sink alone: uniformly distributed in the poloidal (along $\theta$ ) and of gradually increasing strength past $\rho \geq 1$ (see Methods). Cases 1 and 2 are initialized identically; subsequent discrepancies in their temporal evolution is thus direct incidence of the outer boundary condition alone. Case-3 is the gradientdriven twin of Case-2: it tests for the influence of turbulence spreading on the global selforganized state. Background mean gradients for Case-3 are the converged profiles of flux-driven Case-2, other parameters being equal between both computations. Case-3 hovers throughout nonlinear evolution about the statistical state of flux-driven Case-2, effectively prescribing a scale separation between background and fluctuations. For proper comparisons, the same radialpoloidal areas are magnified for each Case: top of the machine [column (a)]; low-field side midplane (b) and bottom regions (c). Reference (circled) positions are systematically displayed in all subplots as well as in Figs. 3 and 4 and supplementary Figs. 3 and 4 at various radial $(\rho=0.9$, 0.96 and 1.02$)$ and poloidal $\left(\theta_{1}\right.$ through $\left.\theta_{5}\right)$ locations.

Symmetry-breaking and free energy injection. We systematically compare a series of three high-resolution numerical experiments (Cases 1 through 3 in Fig.1), mimicking tokamak parameters of the Tore Supra shot \#45511, using the GYSELA framework (14). The coupled gyrokinetic and Poisson equations are time stepped in five dimensions, from core $(\rho=r / a=0)$ to wall ( $\rho=1.3$ ). Cases 1 to 3 only differ through assumptions on forcing or boundary conditions (see Methods). Cases 1 and 2 are flux-driven whilst Case- 3 is the gradient-driven twin of Case-2. Case-1 mimics the experiment, its limiter consisting in a delimited heat and momentum sink through the plasma volume. Conversely, Cases 2 and 3 feature common radially progressive and poloidally-uniform (uniform along $\theta$ ) absorbent boundaries.

Gradient inhomogeneities in the poloidal are pervasive in experiments, though often elusive to diagnose. They may stem (non-exhaustively) from the presence of a limiter, of a radiating $\mathrm{X}$-point or of high-Z impurities. Their determination is a central matter for transport $(15,16)$. Interestingly, a marked difference in edge turbulence (Fig.1) is found upon modification of the outer plasma boundary conditions from limiter to poloidally uniform. Interaction with the limiter in Case-1 onsets and sustains an over-dense poloidal shelf in its vicinity [Fig.2-(c) and (e)] as well as a poloidal distribution of temperature gradients [Fig.2-(d) and (f)] and 
induces a radial electric field well (Fig.2-b), features absent in poloidally homogeneous Cases 2 and 3. As a cold spot, the limiter tends to create radial-poloidal pressure anisotropies in its vicinity, in a manner akin to thermodiffusion described by off-diagonal (density and momentum pinch) terms in the transport matrix. Such a mechanism provides robust free energy injection nearby a localized heat or momentum sink. As such it is possibly generic enough to apply beyond limiter configurations to the vicinity of dissipative $\mathrm{X}$-points as well.

(a)

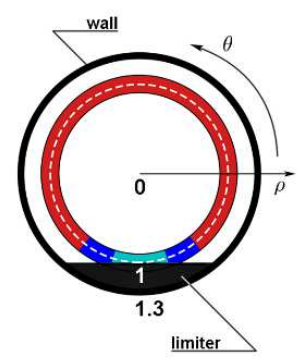

(b) Radial electric field [in $\mathrm{Vm}^{1}$ ]

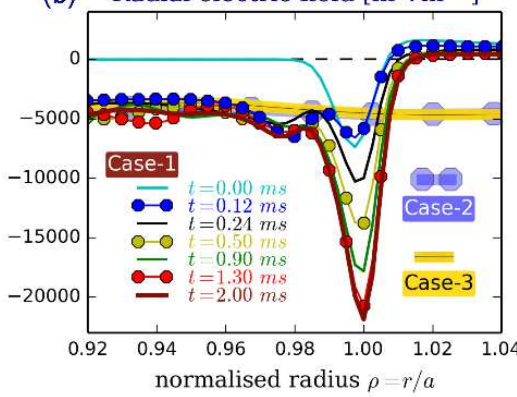

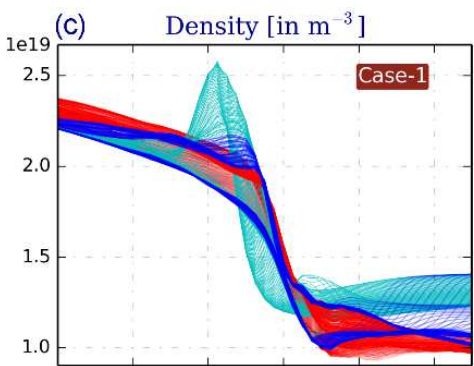

(d) Temperature [in eV]

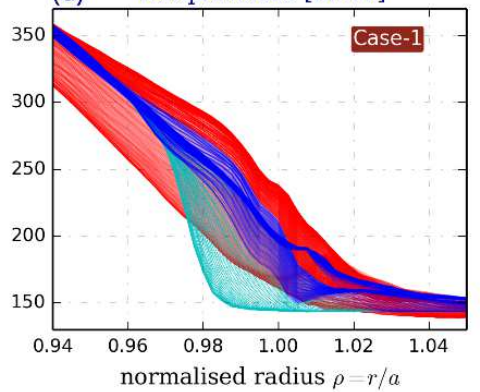

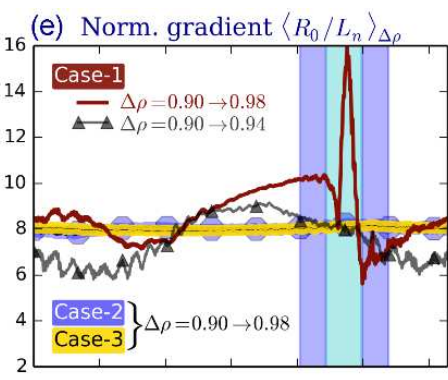

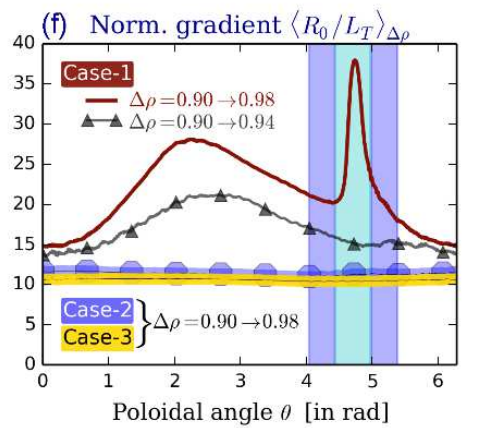

Fig. 2. Spontaneous symmetry breaking from plasma-boundary interplay leads to new free energy sources. Density (c) and temperature (d) profiles are color-coded with poloidal $\theta$ proximity to the limiter (a). Hot plasma particles rapidly stream towards the limiter along field lines as well as through the action of vertical $B \times \nabla B$ magnetic drift (here pointing towards the limiter). This dynamics leads in Case-1 to the onset and sustainment of an over-dense poloidal density shelf (Fig.2-c and e) near the limiter as well as localized temperature gradients (Fig.2-d and f) and a radial electric field well (Fig.2-b, averaged poloidally), absent in poloidally homogeneous Cases 2 and 3. This poloidal anisotropy is especially significant within $5 \%$ of the separatrix, as seen through radial averaging $<.>_{\Delta \rho}$ of equilibrium mean gradients (Fig.2-e and f). Gradient anisotropy decreases in magnitude in the near SOL and poloidally farther from the limiter. Large and anisotropic equilibrium mean gradients in the limited configuration result in locally-enhanced free energy sources for the turbulence in the outermost 5\% layer of the confined edge. Inversely, Cases-2 and 3 display uniform and moderate mean gradients, shown in thick yellow and dotted blue. 
Extensive linear stability analysis of the three Cases has been performed using the initial value framework of the Gyrokinetic Workshop (GKW) code (17) at various radial-poloidal $\left(\rho_{\mathrm{j}}, \theta_{\mathrm{k}}\right)$ edge locations (circles in Fig.1). It leads to the following picture (see Methods): (i) edge profiles in Cases- 2 and 3 are linearly stable to drift-wave, interchange (DWI) and parallel shear flow instabilities past $\rho \geq 0.9$ whilst (ii) a localized region of instability, dominantly of interchange character appears in Case-1 immediately inside the separatrix due to the presence of the limiter, on both sides of it. Electron-scale instabilities or electromagnetic modes, absent in the present modelling may also be active in actual tokamaks and contribute to edge transport. The present work focuses on robust shear flow and ion-scale modes only and thus has the powerful appeal of relative simplicity, whilst being at the fringes of current-day fluxdriven supercomputing capabilities. It allows to define a minimally-relevant set of ingredients that produces transport in the edge consistent with experimental levels and naturally triggers and sustains an edge transport barrier. As such it arguably goes some way towards disentangling the relevant hierarchy of basic mechanisms at play: plasma-boundary interaction builds the local dynamics of the "tail" which helps confinement through edge transport barrier onset and wags the "dog" through scale interplay.

\section{Interfacial contamination unraveled through entropy density budget. Self-advection} (spreading) of patches of turbulence intensity I is quantitatively followed through waveenergy budget (18). The relevant conserved quantity is the negative of the entropy density $(\mathrm{nl})$, which involves the ambient density profile $\mathrm{n}$ and naturally leads to amplification of fluctuations I, as $\mathrm{n}$ decreases in the plasma edge. This "beach effect" is direct consequence of wave-energy density conservation: the fewer the oscillators, the larger the oscillations. The related conservation equation (19): $\mathrm{D} / \mathrm{Dt}(\mathrm{nl})+\nabla \cdot \Gamma_{\mathrm{S}}=\mathrm{S}$ features the wave-energy flux $\Gamma_{S}(r, \theta, t)=1 / 2\left\langle\int d^{3} v\left(v_{E} \cdot \nabla r\right)(\delta f)^{2} / F_{M}\right\rangle_{\varphi}$ as kinetic proxy for spatial turbulence spreading. Here $\mathrm{D} / \mathrm{Dt}$ denotes the turbulent convective derivative, $S$ free energy injection and dissipation mechanisms, ( $\delta$ f) the departure of the full ion distribution function $\bar{F}$ from an ensemble averaged Maxwellian $F_{M}$ reconstructed from the evolving local density and temperature profiles, $\left(\mathbf{v}_{\mathrm{E}} \cdot \nabla \mathbf{r}\right)$ is the radial ExB velocity and $\langle\cdot\rangle_{\varphi}$ toroidal averaging (quantities are further introduced in Methods). 

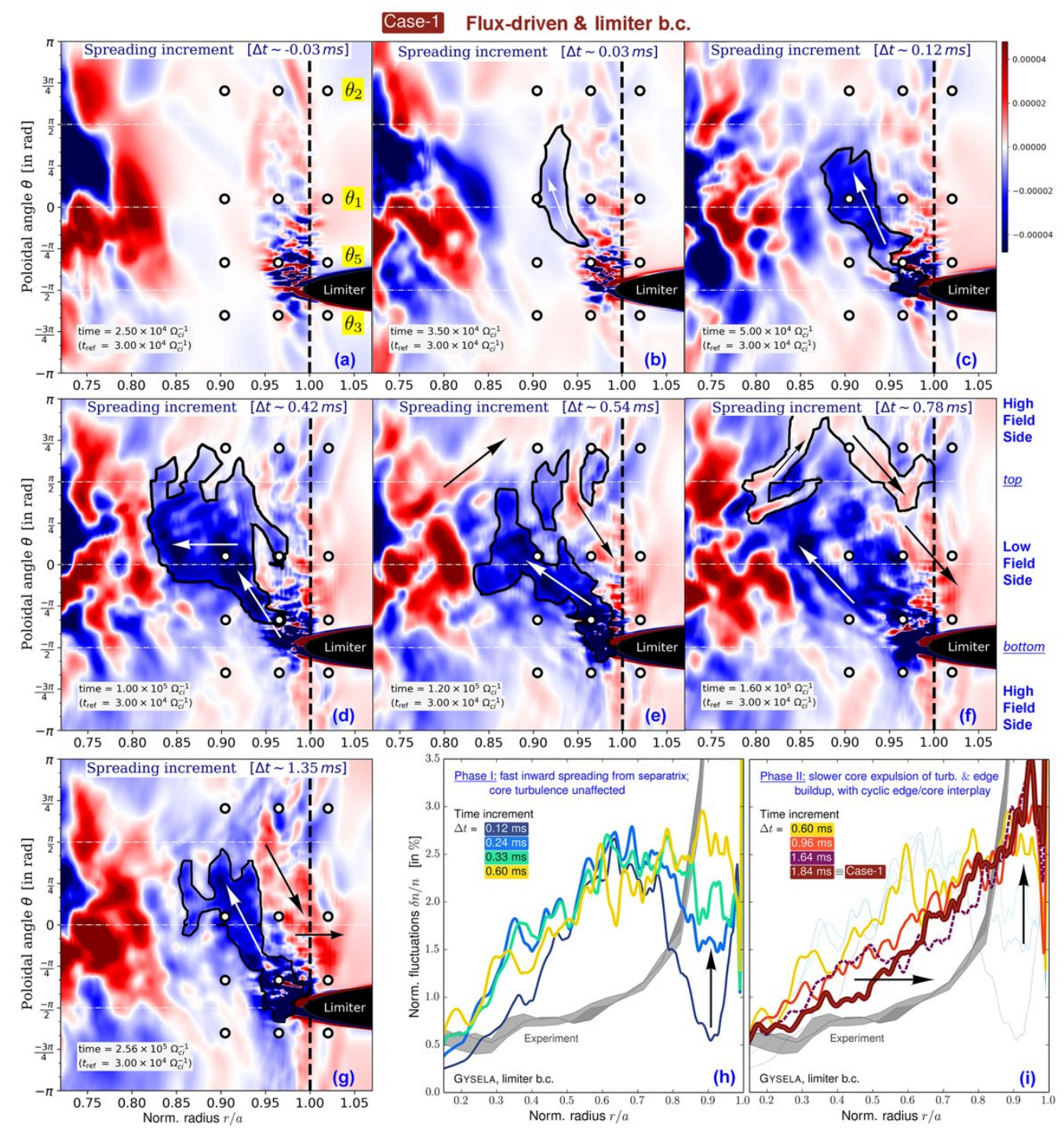

Fig. 3. Spatiotemporal redistribution of induced free energy injection at the plasma-boundary interface (flux-driven Case-1). Subplots (a)-(g): times series of poloidal cross-sections of spreading increments $\Delta S$. Positive (red) increments represent radially-outward fluxes of turbulence intensity; inward fluxes of turbulence intensity are pictured as negative (blue) increments. The limiter is responsible for vigorous mixing in its immediate poloidal (between $\theta_{3}$ and $\left.\theta_{5}\right)$ and radial $(0.95 \leq r / a \leq 1.0)$ vicinity. The drive endures throughout linear Fig.3-(a) and nonlinear evolution $[(b)$ through $(g)]$. The choice of an early reference time $t_{r e f}=30,000 \Omega_{c i}{ }^{-1}$ allows to follow the full unfolding of the spreading sequence. With a later choice for $t_{\text {ref }}$ the early spreading sequence would not appear as clearly; later nonlinear dynamics (Phases II and III) on the other hand would be qualitatively unchanged: cyclic equilibration on the outboard midplane between incoming and outgoing fluxes of turbulence intensity that originate from distinct poloidal regions. Inward spreading (Phase I) is responsible for edge increase of turbulence activity at the outboard midplane [subplot (h)]. As NMsL fills-in, outgoing turbulence activity spreads to the edge [Phase II, subplot (i)], further enhancing relative fluctuation levels in the 
Self-advection of turbulent patches is best apprehended as $\Gamma_{\mathrm{S}}$ increments. Fig.3 displays times series of poloidal cross-sections of spreading increments $\Delta S=\Gamma s(r, \theta, t)-\Gamma_{s}\left(r, \theta, t_{\text {ref }}\right)$ for Case- 1 . Three phases appear: a NMsL devoid of fluctuations (Phase I, subplots (a) through (d)) is clearly visible in the early stages of Case- 1 for all poloidal angles and for $0.85 \leq \mathrm{r} / \mathrm{a} \leq 1$, echoing aforementioned linear stability of underlying profiles. Strong and persistent inward advection of turbulence intensity originates at the edge-limiter boundary (around $\theta=-\pi / 2$ ), propagates (white arrows) radially inwards and poloidally anticlockwise all the way to the top $(\theta=+\pi / 2)$, in about $0.1 \mathrm{~ms}\left[16,700 \Omega_{\mathrm{ci}^{-1}}{ }^{-1}\right.$. From there [Phase II, subplots $\left.(e)-(f)\right]$, outwards radial spreading (black arrows) accompanied by clockwise poloidal motion from the plasma high field side and top regions to the low field side midplane $(\theta=0)$ fills-in NMsL with turbulence in about $0.5 \mathrm{~ms}$. This to and fro redistribution of turbulence intensity bridges the region of free energy injection near the limiter with the upstream confined core. As both regions connect, core turbulence spills over, further enhancing edge fluctuation levels. This complex radialpoloidal dynamics finds an echo in the synthetic reconstructions [Fig.3-(h) and (i)] of the radial profiles of turbulent fluctuations $\delta \mathrm{n} / \mathrm{n}$ around $\theta=0$, as would be measured in Tore Supra. These synthetic profiles are plotted against the actual measurements using fast-swept reflectometry (shaded grey) (20). A dynamic equilibrium (Phase III, subplot (g)), characterized by quasi-periodic relaxations of the edge turbulence is reached at later times (from 1.55ms onward). Bursts of clockwise rotating outgoing patches of turbulence (black arrows in Fig.3-(g) and (i)) equilibrate incoming anticlockwise limiter-borne fluxes of turbulence intensity.
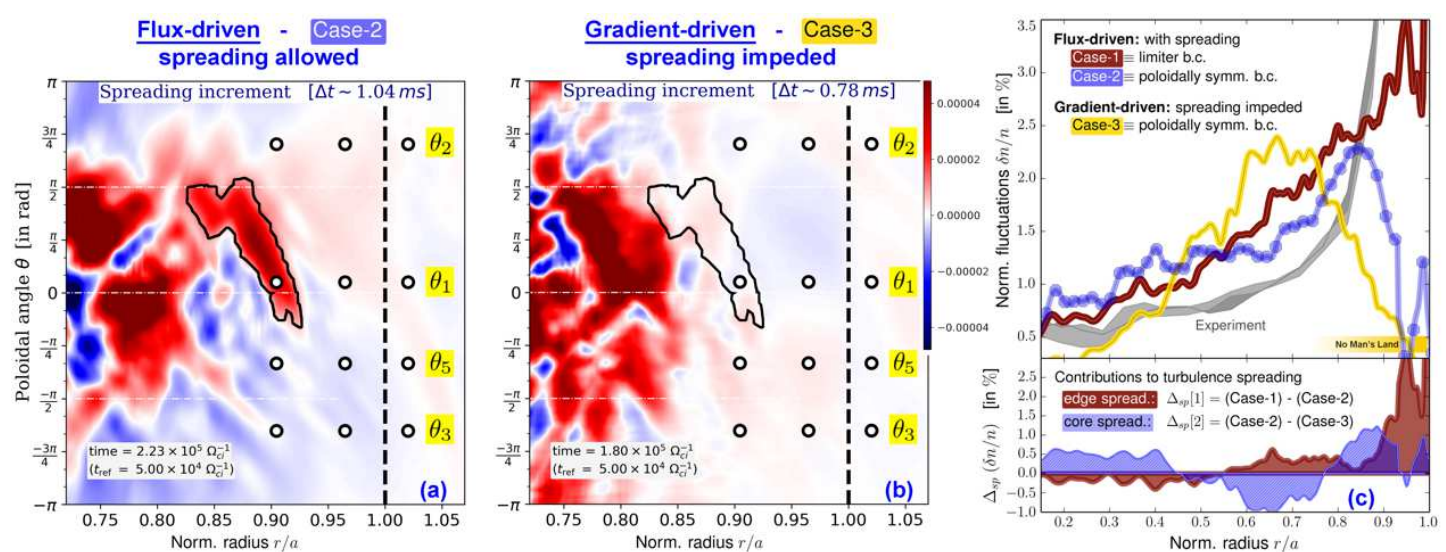

Fig. 4. How forcing and interplay with boundaries impact redistribution of turbulence activity and globally affect transport. Spreading increments for Case-2 (a) and Case-3 (b) at time 
$t_{r e f}=50,000 \Omega_{c i}{ }^{-1}$ when both Cases enter nonlinear regime in the edge. A clear shortfall in NMsL is visible at statistical equilibrium (subplot (c)-top) for Cases 2 and 3 with respect to experimental or to Case-1 fluctuation profiles $\delta n / n$. Differences $\Delta_{\mathrm{sp}}[1]$ and $\Delta_{\mathrm{sp}}[2]$ respectively quantify (subplot (c)-bottom) the importance for turbulent fluctuations of separatrix $\rightarrow$ core and core $\rightarrow$ edge spreading.

Comparatively, Case-2 [Fig.4-(a)] and Case-3 [Fig.4-(b)] equilibrate on faster timescales, with spreading patterns dominantly outwards and reaching equilibrium in about $0.6 \mathrm{~ms}$. A clear shortfall is observed [Fig.4-(c), top] past $0.90 \leq \mathrm{r} / \mathrm{a}$ for Case- 2 and past $0.85 \leq \mathrm{r} / \mathrm{a}$ for Case-3, again expressing linear stability of underlying gradients, uncompensated by fluxes of turbulence activity from distant active (core or boundary) regions. Spreading in gradientdriven Case- 3 is modest, at most about a few local turbulence correlation lengths, as in earlier works (5), and insufficient to explain the required fluctuation levels in the edge.

Normalized contributions $\Delta_{s p}[j]=\delta n / n[j]-\delta n / n[j+1]$ further quantify the weight of spreading [Fig.4-(c), bottom] to overall statistical equilibrium, $\mathrm{j}$ denoting the Case index. Quantity $\Delta_{\mathrm{sp}}[1]$ assesses outside $\rightarrow$ in spreading. Clearly, near-separatrix $\rightarrow$ core contamination of limiter-borne turbulence activity accounts almost in full for the fluctuation dynamics in the outer radial $10 \%$ of the confined plasma. Similarly, $\Delta_{\mathrm{sp}}[2]$ quantifies the weight of inside $\rightarrow$ out spreading of core turbulence intensity towards the edge, amplified by the beach effect. Mean gradients in Cases 2 and 3 being the same by construction, the reversing sign of $\Delta_{\text {sp }}[2]$ illustrates the natural tendency of the flux-driven system with respect to the gradient-driven framework to radially redistribute patches of turbulence intensity, both outward $(0.8 \leq \mathrm{r} / \mathrm{a} \leq 0.92)$ and inward $(\mathrm{r} / \mathrm{a} \leq 0.4)$. Extra spreading in flux-driven approaches, illustrated in subplot (a) by the black contour about $\theta_{1}$ and lack thereof in Case-3 [same contour, subplot (b)] explains the gradient-driven underprediction of fluctuation levels in the deep core $\mathrm{r} / \mathrm{a} \leq 0.45$ and outer edge regions $0.85 \leq \mathrm{r} / \mathrm{a} \leq 0.90$ as well as its overprediction in the intermediate linearlyunstable region $0.55 \leq \mathrm{r} / \mathrm{a} \leq 0.75$.

Causal inference in transport barrier onset. Causality is actively debated in information theory $(21,22)$. We focus here on a nonlinear extension of the Granger causality, using the information-theoretic “ 'Transfer Entropy” (TE) approach $(23,24)$ to question the causal chain of events underpinning transport barrier build-up. TE is generic and allows for a diagrammatic representation of net (directional) information transfer in bivariate time series analysis (25). We apply it to the spontaneous emergence of the stable and localized transport 
barrier which develops at the closed/open field line transition through limiter-induced symmetry-breaking. The sharp radial electric field well at the plasma edge [Fig.2-(b)] is clear signature of it. Application of TE on electric field data from the primitive flux-driven equations opens captivating possibilities to confront with basic mechanisms.

Radial electric field dynamics satisfies a vorticity balance (26-28) which may be systematically derived from the primitive gyrokinetic equation, Eqs.(A-1)-(A-5) in Methods. All terms in Eqs.(A-1) and (A-2) are computed and those with significant magnitudes are displayed in Fig.5 (a). Overall vorticity balance is precisely satisfied (0.9\%), which allows to write at leading order:

$\partial_{t}\left\langle\Omega_{r}\right\rangle+\frac{1}{r} \partial_{r} r\left[\left\langle v_{E r} \Omega_{r}\right\rangle+\left\langle v_{* r} \Omega_{r}\right\rangle-\left\langle v_{* \theta} \frac{\partial_{\theta} E_{r}}{r}\right\rangle\right]=r . h . s \approx 0$

where $\langle\cdot\rangle$ denotes toroidal averaging. Radial vorticity $\left\langle\Omega_{\mathrm{r}}\right\rangle$ (flow shear) thus dominantly evolves through the combined influence of 3 fluxes: the usual ExB (radial) advection of vorticity $\left\langle\mathrm{VEr} \Omega_{\mathrm{r}}\right\rangle$ [hereafter denoted "Reynolds force"] and two seldom discussed mechanisms: diamagnetic (radial) advection of vorticity $\left\langle\mathrm{v}^{*} \mathrm{r} \Omega \mathrm{r}\right\rangle$ and diamagnetic (poloidal) advection of poloidal inhomogeneities of the radial electric field $\left\langle v^{*}{ }_{\theta}\left(\partial_{\theta} E_{r}\right) / r\right\rangle$ [hereafter, "field advection"]. Unexpectedly, in the early stages of radial electric field build-up both latter contributions display magnitudes larger than that of the Reynolds force [Fig.5 (a)]. Dynamical significance to vorticity build-up however does not straightforwardly follow. Systematic TE computations for all pairs of terms in Eq.(1) is performed and the relevant interactions are portrayed in Fig.5-(c), (d) and (e).

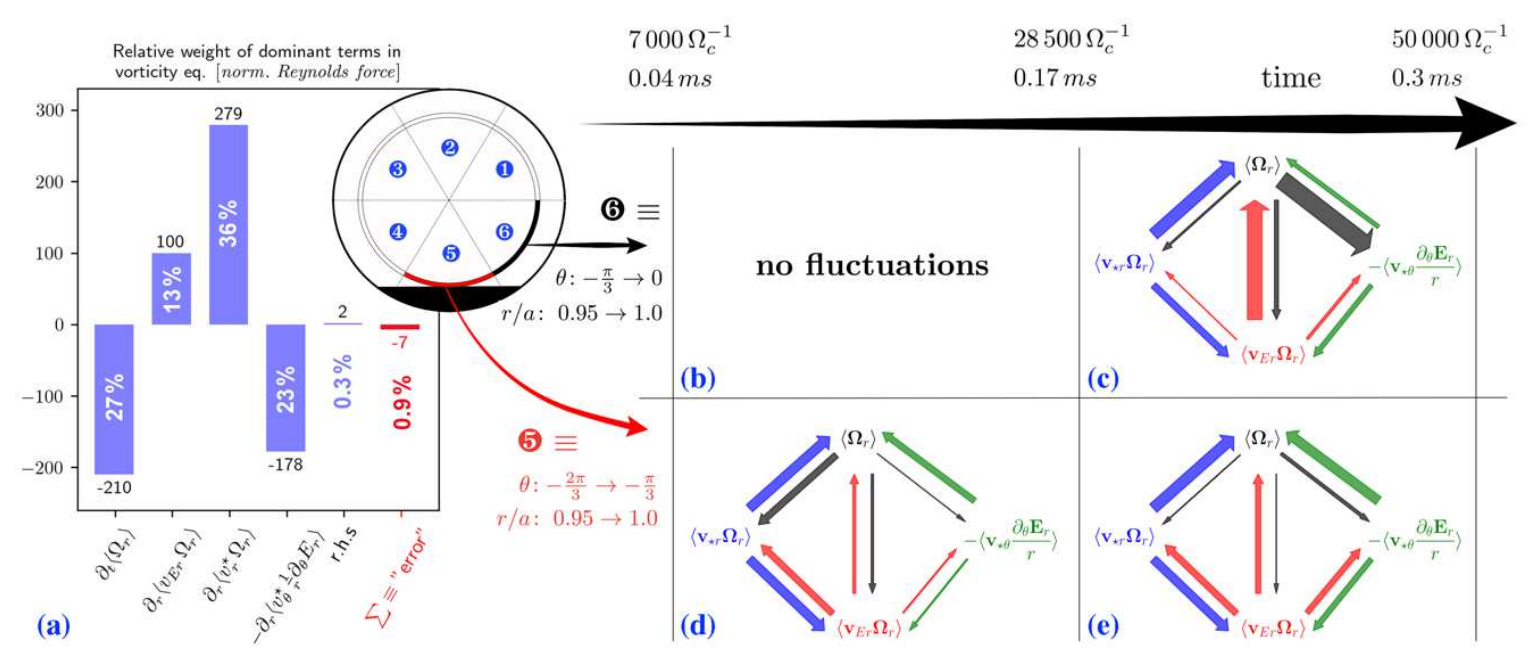

Fig. 5. Causal interactions discriminated with proximity to boundaries in the early stages of barrier build-up (initial $0.3 \mathrm{~ms}$, or $50,000 \Omega_{c^{-1}}$ ), between $0.95 \leq r / a \leq 1.0$ where limiter-induced fluctuations are borne. Subplot (a): Precise test of vorticity balance through systematic 
computation of all terms in Eqs.(A-9) and (A-10). Their relative magnitudes are estimated through radial $(0.95 \leq r / a \leq 1.0)$, poloidal $(0 \leq \theta \leq 2 \pi)$, toroidal $(0 \leq \varphi \leq \pi / 5)$ and temporal $(0 \leq t$ $\Omega_{\mathrm{c}} \leq 50,000$ ) averaging. Their algebraic sum is displayed as "error" (value -7 corresponds to $0.9 \%$ error). All terms in Eq.(A-10) are individually small, with r.h.s denoting their sum. Subplots (b) through (e): the outer corona (0.95 $\leq r / a \leq 1.0)$ of plasma volume is divided in six areas; we especially focus on areas $5(-2 \pi / 3 \leq \theta \leq-\pi / 3)$, centered about the limiter where the radial electric field well originates and area $6(-\pi / 3 \leq \theta \leq 0)$ into which turbulence later spreads. Dominant information transfer from systematic pairwise TE computations is diagrammatically represented for areas $\mathbf{5}$ and $\mathbf{6}$, during two time intervals. During $\Delta t_{1}(0.04 \mathrm{~ms}$ to $0.17 \mathrm{~ms})$, turbulence is confined to area 5 [Fig.5-(d)] and other regions are stable [Fig.5-(b)]. During $\Delta t_{2}$ (0.17ms to $0.3 \mathrm{~ms})$, fluctuations have contaminated area 6 [Fig.5-(c)] whilst keep being produced in area 5 [Fig.5-(e)]. Arrows indicate the direction of information flow and their respective thickness is proportional to the amount of information actually transferred.

Area $\boldsymbol{5}$ is the production region for edge fluctuations and naturally sustains local inhomogeneities in the radial-poloidal plane. Larmor motion in this inhomogeneous background is responsible for persistent diamagnetic currents, whose importance is not commonly stressed. Interestingly here, (i) diamagnetic (radial) advection of vorticity $\left\langle\mathrm{v}^{*} \mathrm{r} \Omega \mathrm{r}\right\rangle$ proves to be at barrier inception the dominant causal agent [Fig.5-(d)] which directly generates vorticity $\left\langle\Omega_{\mathrm{r}}\right\rangle$ and forecasts the Reynolds force $\left\langle\mathrm{vEr}_{\mathrm{Er}} \Omega_{\mathrm{r}}\right\rangle$. This central role of $\left\langle\mathrm{v}^{*}{ }_{\mathrm{r}} \Omega_{\mathrm{r}}\right\rangle$ (ii) endures at later times [Fig.5-(e)] even when the Reynolds force (towards which $\left\langle\mathrm{v}^{*} \mathrm{r} \Omega \mathrm{r}\right\rangle$ keeps transferring information) starts contributing more to vorticity production. The oftexpected dominant transfer mechanism: (iii) Reynolds force causing vorticity production becomes major only at later times $\Delta \mathrm{t}_{2}$ and in area $\boldsymbol{6}$ [Fig.5-(c)], naturally stable without limiter. It has required turbulent fluctuations to have spread there, downstream of production region 5. TE analysis also highlights another (new to our knowledge) mechanism for both vorticity production and shear dissemination: (poloidal) diamagnetic currents contribute to vorticity build-up whilst poloidally propagating radial electric field poloidal inhomogeneities $\left\langle v^{*}{ }_{\theta}\left(\partial_{\theta} E_{r}\right) / r\right\rangle\left[\right.$ Fig.5-(d) and (e)]. Farther downstream [Fig.5-(c)] vortical structures $\left\langle\Omega_{\mathrm{r}}\right\rangle$ transfer information to field advection; this mechanism contributes to expanding the newsprung Er well, initially a localized feature of area $\mathbf{5}$ and making it a poloidally global feature spanning regions $\mathbf{1}$ through $\boldsymbol{0}$.

Summary and outlook. Data-driven entropy-based methods, applicable in principle to a large variety of complex, self-organizing systems have proven fecund in fusion plasmas to 
furthering key aspects of our understanding. Nonlinear destabilization of linearly stable edge occurs through boundary-mediated, localized free energy injection combined with turbulence intensity redistribution (spreading). Hindering of either one of these mechanisms prevents transport barrier onset and leads to a laminar edge, in stark contrast with experiments. Transport properties at the fringes of the confined plasma appear as a case of the tail wagging the dog: outward spreading of bulk core turbulence, though active is subdominant to incoming turbulence penetration from the peripheral near-separatrix region. Fluxes of turbulent intensity in the edge are not merely locally driven by local mean gradients, illustrating a 'nonlocal' character $(29,30)$ of edge-core dynamic coupling. Causal analysis of transport barrier build-up emphasizes the central and somewhat unexpected role of diamagnetic flows $\left(\mathrm{v}^{*}{ }_{\theta}=\partial_{\mathrm{r}} \mathrm{p}_{\perp}\right.$ and $\left.\mathrm{v}_{\mathrm{r}=-}^{*} \partial_{\theta} \mathrm{p}_{\perp} / \mathrm{r}\right)$, shedding light on new or low-keyed mechanisms. This stresses the role of pressure inhomogeneities and finite Larmor radius (FLR) effects in barrier build-up. The importance of the latter could have interesting side effects, foremost on I-mode or H-mode accessibility as different isotopes or different classes of particles (electrons, main ions, energetic particles or impurities) may differently contribute to vorticity (shear) production, affecting access to bifurcated states of enhanced confinement.

\section{References}

1. S. Corrsin, A. L. Kistler. Free-stream boundaries of turbulent flows. Technical Report TN-1244, NACA (1955).

2. A. A. Townsend. The Structure of Turbulent Shear Flow. 2nd Edition, Cambridge University Press, Cambridge (1976).

3. N. H. Brummell, T. L. Clune, J. Toomre. Penetration and overshooting in turbulent compressible convection. The Astrophysical Journal, 570(2):825 (2002).

4. X. Garbet, L. Laurent, A. Samain, J. Chinardet. Radial propagation of turbulence in tokamaks. Nuclear Fusion, 34(7):963-74 (1994).

5. T S Hahm, P H Diamond, Z Lin, K Itoh, S-I Itoh. Turbulence spreading into the linearly stable zone and transport scaling. Plasma Physics and Controlled Fusion, 46(5A):A323 (2004).

6. R. A. Heinonen, P. H. Diamond. Subcritical turbulence spreading and avalanche birth. Physics of Plasmas, 26(3):030701 (2019).

7. Y. Pomeau. Front motion, metastability and subcritical bifurcations in hydrodynamics. Physica D: Non-linear Phenomena, 23(1):3-11 (1986).

8. F. Wagner, G. Becker, K. Behringer, D. Campbell, A. Eberhagen, W. Engelhardt, G. Fussmann, O. Gehre, J. Gernhardt, G. v. Gierke, G. Haas, M. Huang, F. Karger, M. Keilhacker, O. Kluber, M. Kornherr, K. Lackner, G. Lisitano, G. G. Lister, H. M. Mayer, D. Meisel, E. R. Muller, H. Murmann, H. Niedermeyer, W. Poschenrieder, H. Rapp, H. Rohr. Regime of improved confinement and high beta in neutral-beam heated divertor discharges of the ASDEX tokamak. Phys. Rev. Lett., 49(19):1408-1412 (1982). 
9. H. Biglari, P. H. Diamond, P. W. Terry. Influence of sheared poloidal rotation on edge turbulence. Physics of Fluids B, 2(1):1-4 (1990).

10. E. J. Kim P. H. Diamond. Zonal flows and transient dynamics of the L-H transition. Phys. Rev. Lett., 90:185006 (2003).

11. P. C. Liewer. Measurements of microturbulence in tokamaks and comparisons with theories of turbulence and anomalous transport. Nuclear Fusion, 25(5):543 (1985).

12. B. B. Kadomtsev, E. W. Laing. Tokamak Plasma: A Complex Physical System. CRC Press (1993).

13. A. J. Brizard, T. S. Hahm. Foundations of nonlinear gyrokinetic theory. Reviews of Modern Physics, 79(2):421-468 (2007).

14. V. Grandgirard, J. Abiteboul, J. Bigot, T. Cartier-Michaud, N. Crouseilles, G. DifPradalier, Ch. Ehrlacher, D. Esteve, X. Garbet, Ph. Ghendrih, G. Latu, M. Mehrenberger, C. Norscini, Ch. Passeron, F. Rozar, Y. Sarazin, E. Sonnendrucker, A. Strugarek, D. Zarzoso. A 5d gyrokinetic full-global semi-lagrangian code for flux-driven ion turbulence simulations. Computer Physics Communications, 207:35 - 68 (2016).

15. C Angioni, P Helander. Neoclassical transport of heavy impurities with poloidally asymmetric density distribution in tokamaks. Plasma Physics and Controlled Fusion, 56(12):124001 (2014).

16. P Donnel, X Garbet, Y Sarazin, Y Asahi, F Wilczynski, E Caschera, G DifPradalier, P Ghendrih, C Gillot. Turbulent generation of poloidal asymmetries of the electric potential in a tokamak. Plasma Physics and Controlled Fusion, 61(1):014003 (2018).

17. A. G. Peeters, Y. Camenen, F. J. Casson, W. A. Hornsby, A. P. Snodin, D. Strintzi, G. Szepesi. The nonlinear gyro-kinetic flux tube code GKW. Computer Physics Communications, 180(12):2650-2672 (2009).

18. N. Mattor, P. H. Diamond. Drift wave propagation as a source of plasma edge turbulence. Phys. Rev. Lett., 72:486-489 (1994).

19. O. D. Gurcan, P. H. Diamond, X. Garbet, V. Berionni, G. Dif-Pradalier, P. Hennequin, P. Morel, Y. Kosuga L. Vermare. Transport of radial heat flux and second sound in fusion plasmas. Physics of Plasmas, 20(2):022307 (2013).

20. F. Clairet, S. Heuraux, C. Bottereau, D. Molina, L. Ducobu, F. Leroux, A. Barbuti. Fast sweeping reflectometry upgrade on tore supra. Review of Scientic Instruments, 81(10):10D903 (2010).

21. Katerina Hlavackova-Schindler, Milan Palus, Martin Vejmelka, Joydeep Bhattacharya. Causality detection based on information-theoretic approaches in time series analysis. Physics Reports, 441(1):1-46 (2007).

22. C.W.J. Granger. Testing for causality: A personal viewpoint. Journal of Economic Dynamics and Control, 2:329-352 (1980).

23. Thomas Schreiber. Measuring information transfer. Phys. Rev. Lett., 85:461-464 (2000).

24. B.Ph. van Milligen, G. Birkenmeier, M. Ramisch, T. Estrada, C. Hidalgo, A. Alonso. Causality detection and turbulence in fusion plasmas. Nuclear Fusion, 54(2):023011 (2014).

25. G. Dif-Pradalier, Ph. Ghendrih, Y. Sarazin, X. Garbet, V Grandgirard, Y. Munschy, R. Varennes, L. Vermare, Y. Camenen, F. Widmer. Interplay between core, edge and scrape-off layer in turbulent magnetized plasmas: Lessons learnt from an information 
theoretic measure, the "transfer entropy" to assess causality in nonlinear plasma dynamics. FEC 2020 - IAEA Fusion Energy Conference (2021).

26. J. Abiteboul, X. Garbet, V. Grandgirard, S. J. Allfrey, Ph. Ghendrih, G. Latu, Y. Sarazin, A. Strugarek. Conservation equations and calculation of mean flows in gyrokinetics. Physics of Plasmas, 18(8):082503 (2011).

27. Ajay C. J., Stephan Brunner, Ben McMillan, Justin Ball, Julien Dominski, Gabriele Merlo. How eigenmode self-interaction affects zonal flows and convergence of tokamak core turbulence with toroidal system size. Journal of Plasma Physics, 86(5):905860504 (2020).

28. Y Sarazin, G Dif-Pradalier, X Garbet, P Ghendrih, A Berger, C Gillot, V Grandgirard, K Obrejan, R Varennes, L Vermare, T Cartier-Michaud. Key impact of phase dynamics and diamagnetic drive on Reynolds stress in magnetic fusion plasmas. Plasma Physics and Controlled Fusion, 63(6):064007 (2021).

29. G. Dif-Pradalier, P. H. Diamond, V. Grandgirard, Y. Sarazin, J. Abiteboul, X. Garbet, Ph. Ghendrih, A. Strugarek, S. Ku, C. S. Chang. On the validity of the local diffusive paradigm in turbulent plasma transport. Phys. Rev. E, 82(2):025401(R) (2010).

30. K. Ida, Z. Shi, H.J. Sun, S. Inagaki, K. Kamiya, J.E. Rice, N. Tamura, P.H. Diamond, G. Dif-Pradalier, X.L. Zou, K. Itoh, S. Sugita, O.D. Gurcan, T. Estrada, C. Hidalgo, T.S. Hahm, A. Field, X.T. Ding, Y. Sakamoto, S. Oldenburger, M. Yoshinuma, T. Kobayashi, M. Jiang, S.H. Hahn, Y.M. Jeon, S.H. Hong, Y. Kosuga, J. Dong, S.-I. Itoh. Towards an emerging understanding of non-locality phenomena and non-local transport. Nuclear Fusion, 55(1):013022 (2015).

31. Y. Sarazin, V. Grandgirard, J. Abiteboul, S. Allfrey, X. Garbet, Ph. Ghendrih, G. Latu, A. Strugarek, G. Dif-Pradalier, P.H. Diamond, S. Ku, C.S. Chang, B.F. McMillan, T.M. Tran, L. Villard, S. Jolliet, A. Bottino, P. Angelino. Predictions on heat transport and plasma rotation from global gyrokinetic simulations. Nuclear Fusion, 51(10):103023 (2011).

32. G. Dif-Pradalier, G. Hornung, X. Garbet, Ph. Ghendrih, V. Grandgirard, G. Latu, Y. Sarazin. The ExB staircase of magnetised plasmas. Nuclear Fusion, 57(6):066026 (2017).

33. B. F. McMillan, S. Jolliet, T. M. Tran, L. Villard, A. Bottino, P. Angelino. Long global gyrokinetic simulations: Source terms and particle noise control. Physics of Plasmas, 15(5):052308 (2008).

34. A. Paredes, H. Bufferand, G. Ciraolo, F. Schwander, E. Serre, P. Ghendrih, P. Tamain. A penalization technique to model plasma facing components in a tokamak with temperature variations. Journal of Computational Physics, 274:283-298 (2014).

35. E. Caschera, G. Dif-Pradalier, Ph. Ghendrih, V. Grandgirard, Y. Asahi, N. Bouzat, P. Donnel, X. Garbet, G. Latu, C. Passeron, Y. Sarazin. Immersed boundary conditions in global, flux-driven, gyrokinetic simulations. Journal of Physics: Conference Series, 1125:012006 (2018).

36. Nicola D'Angelo. Kelvin-Helmholtz instability in a fully ionized plasma in a magnetic field. The Physics of Fluids, 8(9):1748-1750 (1965).

\section{Supplementary information}

See companion PDF file 
Raw data generated by the GYSELA code is available from the corresponding author upon request.

\section{Code availability}

The GYSELA suite of codes used for this article is available upon request via the GitHub repository https://gyselax.github.io

\section{Acknowledgements}

The authors acknowledge stimulating discussions with P.H. Diamond and participants at the 2019 and 2021 "Festival de Théorie" in Aix-en-Provence. This work has been carried out within the framework of the EUROfusion Consortium and was supported by the EUROfusion Theory and Advanced Simulation Coordination (E-TASC) initiative under the TSVV (Theory, Simulation, Verification and Validation) "L-H transition and pedestal physics" project (2019-2020) and TSVV "Plasma Particle/Heat Exhaust: Gyrokinetic/Kinetic Edge Codes" (2021-2025). It has also received funding from the Euratom research and training program. The authors gratefully acknowledge funding from the European Commission Horizon 2020 research and innovation program under Grant Agreement No. 824158 (EoCoE-II). The views and opinions expressed herein do not necessarily reflect those of the European Commission. We acknowledge PRACE for awarding us access to Joliot-Curie at GENCI@CEA, France and MareNostrum at Barcelona Supercomputing Center (BSC), Spain.

\section{Contributions}

G.D-P., Ph.Gh. and E.C. designed and implemented the penalization technique, G.D-P. performed the GYSELA computations, wrote the Transfer Entropy method and performed the analysis. V.G. is the main developer of the the GYSELA code. L.V and F.C. provided the experimental data. F.W. and Y.C. performed the linear GKW analysis. G.D-P., Y.S., X.G. and Ph.Gh. derived and discussed the vorticity equation. G.D-P. wrote the manuscript. All authors discussed the results and commented on the paper.

\section{Competing interests}

The authors declare no competing interests.

\section{Corresponding author}

Guilhem Dif-Pradalier guilhem.dif-pradalier@cea.fr 


\section{Supplementary Files}

This is a list of supplementary files associated with this preprint. Click to download.

- 21TailDogNatPhyssupplementary.pdf 